\title{
PRODUÇÃO CIENTÍFICA SOBRE CENTRO CIRÚRGICO EM TESES E DISSERTAÇÕES: ESTUDO BIBLIOMÉTRICO
}

\author{
Scientific production about surgical center in theses and dissertations: a bibliometric study
}

Producción científica sobre centro quirúrgico en tesis y disertaciones: estudio bibliométrico

Alexmália Fiorini da Costa Balonecker ${ }^{1}$ (D) Cintia Silva Fassarella ${ }^{2 *}$ (D), Priscilla Alfradique de Souza ${ }^{3}$ (D), Ricardo de Oliveira Meneses ${ }^{4}$, Aline Affonso Luna² (D), Mariana Brito de Souza Nunes ${ }^{1}$ (D), Rosilene Alves Ferreira' (D)

RESUMO: Objetivo: Identificar a produção acadêmica advinda de dissertações e teses sobre centro cirúrgico de enfermeiros que concluíram mestrados acadêmicos e doutorados no Brasil. Método: Estudo bibliométrico, descritivo e retrospectivo, com pesquisa documental em três bases de dados brasileiras, recorrendo às publicações de 11 instituições de ensino superior, no período de 2005 a 2016. Resultados: Identificou-se um total de 40 produções acadêmicas. Dessas, 13 são teses (32,5\%) e 27 são dissertações (67,5\%). Os anos com maior publicação (17,5\%) foram 2009 e 2013. A Universidade de São Paulo e a Universidade de São Paulo Ribeirão Preto são as instituições com maior representatividade de estudos, com 32,5 e 25,0\%, respectivamente. A abordagem metodológica mais adotada pelos pesquisadores foi a quantitativa $(40,0 \%)$ e a temática principal tratou da assistência perioperatória (60,0\%). Conclusão: A produção científica nessa área tem se apresentado incipiente, quando comparada ao número total de publicações de pós-graduação stricto sensu de enfermagem no cenário nacional. Entretanto, o que se tem publicado está voltado para assistência hospitalar, demonstrando correlação dos estudos com a prática de enfermagem perioperatória.

Palavras-chave: Centros cirúrgicos. Período intraoperatório. Enfermagem de centro cirúrgico. Bibliometria.

ABSTRACT: Objective: To identify the academic production coming from dissertations and theses about surgical centers by nurses who have completed master's degrees and doctorates in Brazil. Method: Bibliometric, descriptive and retrospective study, with documentary research in three Brazilian databases, using the publications of 11 higher education institutions, from 2005 to 2016. Results: A total of 40 academic productions were identified. Of these, 13 are theses (32.5\%) and 27 are dissertations (67.5\%). The years with the most publications (17.5\%) were 2009 and 2013. The University of São Paulo and the University of São Paulo - Ribeirão Preto are the institutions with the highest number of studies, with 32.5 and $25.0 \%$, respectively. The methodological approach most adopted by the researchers was the quantitative one (40.0\%) and the main theme dealt with perioperative care (60.0\%). Conclusion: The scientific production in this area has presented incipient, when compared to the total number of post-graduation publications stricto sensu on nursing in the national scenario. However, what has been published is aimed at hospital care, showing correlation of the studies with the practice of perioperative nursing. Keywords: Surgicenters. Intraoperative period. Operating room nursing. Bibliometrics.

RESUMEN: Objetivo: Identificar la producción académica proveniente de disertaciones y tesis sobre centros quirúrgicos por enfermeras que hayan completado maestrías y doctorados en Brasil. Método: estudio bibliométrico, descriptivo y retrospectivo, con investigación documental en tres bases de datos brasileñas, utilizando las publicaciones de 11 instituciones de educación superior, de 2005 a 2016. Resultados: Se identificaron un total de 40 producciones académicas. De estas, 13 son tesis (32,5\%) y 27 son disertaciones (67,5\%). Los años con la mayor cantidad de publicaciones (17,5\%) fueron 2009 y 2013. La Universidad de São Paulo y la Universidad de São Paulo - Ribeirão Preto son las instituciones con mayor número de estudios, con 32,5 y 25,0\%, respectivamente. El enfoque metodológico más adoptado por los investigadores fue el cuantitativo $(40,0 \%)$ y el tema principal fue el cuidado perioperatorio (60,0\%). Conclusión: La producción científica en esta área ha presentado incipientes, en comparación con el número total de publicaciones de postgrado en stricto sensu sobre enfermería en el escenario nacional. Sin embargo, lo que se ha publicado está dirigido a la atención hospitalaria y muestra la correlación de los estudios con la práctica de la enfermería perioperatoria. Palabras-clave: Centros quirúrgicos. Periodo intraoperatorio. Enfermería de quirófano. Bibliometría.

'Enfermeira; residente de Enfermagem no Centro Cirúrgico, Centro de Material e Esterilização do Hospital Universitário Pedro Ernesto (HUPE), Universidade do Estado do Rio de Janeiro (UERJ) Rio de Janeiro (RJ), Brasil.

2Doutora em Enfermagem; professora assistente da UERJ e professora adjunta da Universidade do Grande Rio (Unigranrio) - Rio de Janeiro (RJ), Brasil.

${ }^{3}$ Doutora em Enfermagem; professora assistente da UERJ - Rio de Janeiro (RJ), Brasil.

4Mestre em Enfermagem; professor assistente da UERJ - Rio de Janeiro (RJ), Brasil.

*Autora correspondente: cintiafassarella@gmail.com

Recebido: 27/03/2018 - Aprovado: 24/06/2018

DOI: $10.5327 / Z 1414-4425201800040008$

| $212 \mid$

REV. SOBECC, SÃO PAULO. OUT./DEZ. 2018; 23(4): 212-217 


\section{INTRODUÇÃO}

O centro cirúrgico (CC) é um campo complexo e de grande interface no contexto hospitalar, constituído por várias áreas interdependentes para permitir ótimas condições à realização do ato anestésico-cirúrgico. Em circunstâncias assépticas ideais, o CC tem por objetivo promover segurança para o paciente e estrutura e conforto para a equipe que o assiste ${ }^{1,2}$.

Para o CC se tornar um setor complexo, percorreu-se uma história de grandes mudanças, inovações tecnológicas e aquisição de competência profissional. No início do século XIX, muitos fatores dificultavam o acontecimento e o percurso de uma cirurgia: instrumentais precários, matéria-prima imprópria e planta física inadequada. No decorrer do século XXI, com o avanço da anestesia, os procedimentos cirúrgicos foram se tornando cada vez mais especializados e precisos ${ }^{3}$.

Com a evolução histórica das cirurgias, foi necessário o desenvolvimento de recursos humanos especializados inseridos nesse cenário. A enfermagem, por compor a equipe do CC, é essencial para o desenvolvimento da assistência integral, de qualidade e ainda responsável por manter um ambiente seguro, confortável e limpo ${ }^{4}$.

A enfermagem perioperatória começou informalmente, com a atuação do profissional com os "cirurgiões-barbeiros", na limpeza de material e na estabilização de pacientes. A consolidação da atuação do enfermeiro cirúrgico foi fortemente influenciada pela criação da atual Association of periOperative Registered Nurses (AORN), cuja missão consiste em unificar os enfermeiros perioperatórios, proporcionar educação e recomendar padrões para o cuidado do paciente cirúrgico ${ }^{5}$.

Já no Brasil, essa missão é exercida pela Associação Brasileira de Enfermeiros de Centro Cirúrgico, Recuperação Anestésica e Centro de Material e Esterilização (SOBECC). Com o avanço da atuação dessas associações, alguns conceitos foram desenvolvidos, bem como a preocupação com a atuação científica do enfermeiro ${ }^{5}$.

Para consolidar uma assistência de enfermagem de qualidade, as evidências científicas são indispensáveis. Uma das formas de identificar as produções científicas disponíveis e de se obter um consenso informativo é a pesquisa bibliométrica. Esta possibilita contagens relevantes, como a de produção por região, temporalidades das publicações, busca por área de conhecimento, literatura relacionada à citação do estudo e fator de impacto de uma publicação científica ${ }^{6,7}$.

A bibliometria é caracterizada como a métrica que trabalha com documentos, artigos, autores, entre outros eventos. Ela não se preocupa somente com o aspecto quantitativo; preocupa-se também em verificar a relevância e o impacto de autores, periódicos, instituições, grupos ou países nas mais diversas áreas do saber ${ }^{8}$.

Os estudos bibliométricos estão alicerçados em um conjunto de leis e princípios empíricos, provenientes da ciência da informação, cuja finalidade é investigar os aspectos quantitativos da produção, da disseminação e do uso da informação disponível e registrada, contribuindo, dessa forma, para a avaliação do estado atual da ciência, assim como do gerenciamento da pesquisa. É por meio dos seus indicadores bibliométricos que o pesquisador tem a possibilidade de traçar um perfil do mundo científico ${ }^{8,9}$.

Diante da importância da assistência de enfermagem ao paciente cirúrgico e do aumento de procedimentos cirúrgicos devido a doenças, traumas e expectativa de vida, considerou-se necessário conhecer as produções científicas sobre CC nesse contexto, uma vez que ela ainda é vista como uma área desconhecida. Complementarmente, pondera-se que a produção de conhecimento divulgada por dissertações de mestrado acadêmico (DMA) e teses de doutorado (TD) pode contribuir para o desenvolvimento da qualificação do ensino e da prática de enfermagem.

Nesse sentido, questiona-se: qual é a produção científica acadêmica sobre CC advinda de dissertações e teses de enfermeiros que concluíram mestrados acadêmicos e doutorados no Brasil? Quais são os principais objetos de estudos abordados por enfermeiros em CC que concluíram mestrado acadêmico e doutorado no Brasil? Quais são os tipos de estudos mais utilizados por esses enfermeiros que concluíram mestrado e doutorado no Brasil?

\section{OBJETIVOS}

\section{Objetivo geral}

Identificar a produção acadêmica sobre CC advinda de dissertações e teses de enfermeiros que concluíram mestrados acadêmicos e doutorados no Brasil.

\section{Objetivo específico}

Identificar os principais objetos de estudo contemplados nas dissertações de mestrado acadêmico e teses de doutorado, a metodologia utilizada, assim como as universidades que produziram esses estudos. 


\section{MÉTODO}

Trata-se de um estudo bibliométrico, descritivo e retrospectivo, realizado mediante pesquisa documental. Esse método permite avaliação objetiva da produção científica, sendo utilizado em diversas áreas do conhecimento científico. A pesquisa bibliométrica, como técnica, compreende a leitura, a seleção e o arquivo dos tópicos de interesse para a pesquisa em questão, com a finalidade de conhecer as contribuições científicas que se efetuaram sobre determinado assunto?.

As fontes de pesquisa foram produzidas com a temática CC, realizadas por enfermeiros e registradas em três bases de dados brasileiras. Estas foram o banco de teses e dissertações da Coordenação de Aperfeiçoamento de Pessoal de Nível Superior (CAPES), na Plataforma Sucupira, a Biblioteca Digital Brasileira de Teses e Dissertações Digitais (BDTD), do Instituto Brasileiro de Informação em Ciência e Tecnologia (IBICT), e o Centro de Estudos e Pesquisas em Enfermagem da Associação Brasileira de Enfermagem (CEPEn-ABEn).

Foram utilizados os Descritores em Ciências da Saúde (DeCS): centros cirúrgicos, período intraoperatório, bibliometria e enfermagem para as duas primeiras bases. Na CEPEnABEn, foi realizada leitura individual dos catálogos disponíveis por ano, sendo realizada a busca apenas por um pesquisador.

A pesquisa abrangeu DMA e TD concluídas no período de 2005 a 2016, provenientes de universidades com nota mínima 5. Tal delimitação de período se deu em função da avaliação quadrienal do Qualis pela CAPES, que tem por objetivo acompanhar e aperfeiçoar os processos de avaliação dos programas de pós-graduação stricto sensu (mestrado e doutorado). Assim, todos esses programas são submetidos a uma criteriosa avaliação periódica, recebendo notas na seguinte escala: 1 e 2 indicam notas mínimas - os que recebem essas notas têm canceladas as autorizações de funcionamento e o reconhecimento dos cursos de mestrado e/ ou doutorado por ele oferecidos; 3 aponta desempenho regular, atendendo ao padrão mínimo de qualidade; 4 é considerado uma boa performance; e 5 é a nota máxima para programas apenas com mestrado com um bom desempenho. As notas 6 e 7 indicam atuação equivalente ao alto padrão internacional, atribuídas exclusivamente aos cursos de doutorado. O Ministério da Educação, por meio do Conselho Nacional de Educação (CNE), reconhece os resultados da avaliação dos cursos novos e da análise recorrente da CAPES.

Para a busca nos bancos de dados, adotaram-se como critérios de inclusão as produções de DMA e TD com temas relacionados ao transoperatório, estudos produzidos por enfermeiros das universidades com notas CAPES a partir de 5 e publicações no período de 2005 a 2016. Os parâmetros de exclusão utilizados foram as TD e DMA que não estavam disponíveis na íntegra para leitura, dissertações de mestrado profissional e temas associados ao centro obstétrico.

O presente estudo foi realizado por meio das seguintes etapas: identificação do tema e da questão norteadora; estabelecimento dos critérios predefinidos; busca e seleção da produção científica na base de dados no período de março a julho de 2017; e tabulação dos dados em planilha usando o programa Microsoft Office Excel 2016. As variáveis delimitadas no estudo foram tratadas por meio da análise quantitativa descritiva.

\section{RESULTADOS}

Foram identificadas as seguintes informações quantitativas em relação à produção acadêmica: 3.720 na plataforma CAPES, 4.103 no CEPEn e 839 na plataforma BDTD, totalizando 8.662 produções acadêmicas de DMA e TD.

Foram excluídas 8.622 produções por não contemplarem os critérios de inclusão preestabelecidos. Ao fim, foram analisadas 40 produções acadêmicas que atenderam aos critérios de inclusão adotados para o presente estudo (Tabela 1). Dessas produções, 13 são TD (32,5\%) e 27 são DMA (67,5\%).

As instituições de ensino superior com TD e DMA que se enquadraram nos critérios de inclusão do presente estudo foram: Universidade Federal de Minas Gerais (UFMG), Universidade Federal do Rio Grande do Sul (UFRGS), Universidade Federal de Santa Catarina (UFSC), Universidade Federal do Rio de Janeiro (UFRJ), Universidade Federal do Rio Grande (UFRG), Universidade Federal do Ceará (UFC), Universidade Estadual de Campinas (UNICAMP), Universidade de São Paulo (USP), Universidade de São Paulo — Ribeirão Preto (USP-RP), Universidade Federal de São Paulo (UNIFESP) e Universidade do Estado do Rio de Janeiro (UERJ). Destaca-se um predomínio de publicações das instituições de ensino da região Sudeste (Tabela 2).

Quanto ao tipo de abordagem metodológica, verifica-se que as produções acadêmicas quantitativas são as mais realizadas $(40,0 \%)$, seguidas pelas qualitativas $(30,0 \%)$, conforme Tabela 3 . Cinco estudos apresentam abordagem quanti-qualitativa, quatro utilizaram o método de revisão integrativa de literatura e um abordou a pesquisa com a revisão sistemática de literatura.

Quando os estudos não informavam explicitamente em seu resumo o tipo de abordagem metodológica, utilizou-se a 
relação entre objetivo, instrumento de coleta de dados, análise e resultados para compreender o tratamento utilizado; isso aconteceu principalmente nas produções de abordagem quanti-qualitativas.

Referente aos objetos de estudo abordados pelas produções científicas em TD e DMA, após análise individual de cada produção, categorizaram-se quatro áreas:

Tabela 1. Produção científica de teses de doutorado e dissertações de mestrado acadêmico sobre centro cirúrgico por ano.

\begin{tabular}{|c|c|c|c|c|c|c|}
\hline \multirow{2}{*}{ Ano } & \multicolumn{2}{|c|}{ Teses } & \multicolumn{2}{c|}{ Dissertações } & \multicolumn{3}{c|}{ Total } \\
\cline { 2 - 7 } & $\mathbf{N}$ & $\%$ & $\mathbf{N}$ & $\%$ & $\mathbf{N}$ & $\%$ \\
\hline 2016 & 1 & 2,5 & 1 & 2,5 & 2 & 5,0 \\
\hline 2015 & 1 & 2,5 & 1 & 2,5 & 2 & 5,0 \\
\hline 2014 & 3 & 7,5 & 2 & 5,0 & 5 & 12,5 \\
\hline 2013 & 1 & 2,5 & 6 & 15,0 & 7 & 17,5 \\
\hline 2012 & 1 & 2,5 & 1 & 2,5 & 2 & 5,0 \\
\hline 2011 & 2 & 5,0 & 3 & 7,5 & 5 & 12,5 \\
\hline 2010 & 0 & 0,0 & 2 & 5,0 & 2 & 5,0 \\
\hline 2009 & 2 & 5,0 & 5 & 12,5 & 7 & 17,5 \\
\hline 2008 & 1 & 2,5 & 3 & 7,5 & 4 & 10,0 \\
\hline 2007 & 0 & 0,0 & 1 & 2,5 & 1 & 2,5 \\
\hline 2006 & 0 & 0,0 & 1 & 2,5 & 1 & 2,0 \\
\hline 2005 & 1 & 2,5 & 1 & 2,5 & 2 & 5,0 \\
\hline Total & 13 & 32,5 & 27 & 67,5 & 40 & 100 \\
\hline
\end{tabular}

Tabela 2. Regiões e instituições mais representadas nas teses e dissertações no período de 2005 a 2016.

\begin{tabular}{|c|c|c|c|c|c|c|c|}
\hline \multirow{2}{*}{ Região } & \multirow{2}{*}{ Instituição } & \multicolumn{2}{|c|}{ Teses } & \multicolumn{2}{|c|}{ Dissertações } & \multicolumn{2}{|c|}{ Total } \\
\hline & & $\mathbf{N}$ & $\%$ & $\mathbf{N}$ & $\%$ & $\mathbf{N}$ & $\%$ \\
\hline \multirow{7}{*}{ Sudeste } & USP & 5 & 12,5 & 8 & 20,0 & 13 & 32,5 \\
\hline & USP-RP & 5 & 12,5 & 5 & 12,5 & 10 & 25,0 \\
\hline & UFMG & 1 & 2,5 & 2 & 5,0 & 03 & 7,5 \\
\hline & UFRJ & 1 & 2,5 & 3 & 7,5 & 04 & 10,0 \\
\hline & UNICAMP & 1 & 2,5 & 0 & 0,0 & 01 & 2,5 \\
\hline & UNIFESP & 0 & 0,0 & 2 & 5,0 & 02 & 5,0 \\
\hline & UERJ & 0 & 0,0 & 4 & 10,0 & 04 & 10,0 \\
\hline \multirow{2}{*}{ Sul } & UFRGS & 0 & 0,0 & 2 & 5,0 & 02 & 5,0 \\
\hline & UFSC & 0 & 0,0 & 1 & 2,5 & 01 & 2,5 \\
\hline Total & & 13 & 32,5 & 27 & 67,5 & 40 & 100 \\
\hline
\end{tabular}

USP: Universidade de São Paulo; USP-RP: Universidade de São Paulo campus Ribeirão Preto; UFMG: Universidade Federal de Minas Gerais; UFRJ: Universidade Federal do Rio de Janeiro; UNICAMP: Universidade Estadual de Campinas; UNIFESP: Universidade Federal de São Paulo; UERJ: Universidade do Estado do Rio de Janeiro; UFRGS: Universidade Federal do Rio Grande do Sul; UFSC: Universidade Federal de Santa Catarina.
1. Assistência transoperatória;

2. Saúde do trabalhador;

3. Custos;

4. Gestão em enfermagem (Tabela 4).

Tabela 3. Produção científica de teses de doutorado e dissertações de mestrado acadêmico sobre centro cirúrgico, segundo métodos de pesquisa.

\begin{tabular}{|l|l|l|}
\hline Variáveis & N & $\%$ \\
\hline $\begin{array}{l}\text { Estudos qualitativos } \\
\text { Descritivo, exploratório }\end{array}$ & 8 & 20,0 \\
\hline $\begin{array}{l}\text { Estudo de caso com entrevista } \\
\text { semiestruturada }\end{array}$ & 1 & 2,5 \\
\hline Etnometodológico & 1 & 2,5 \\
\hline Estudo clínico & 1 & 2.5 \\
\hline Teoria Fundamentada dos Dados (TFD) & 1 & 2,5 \\
\hline Estudos quantitativos & & \\
\hline Transversal, descritivo, exploratório & 7 & 17,5 \\
\hline Retrospectivo & 1 & 2,5 \\
\hline Observacional & 2 & 5,0 \\
\hline Não experimental, correlacional, prospectivo & 2 & 5,0 \\
\hline Comparativo & 1 & 2,5 \\
\hline Experimental & 2 & 5,0 \\
\hline Estudo de caso, descritivo, exploratório, & 1 & 2,5 \\
\hline correlacional, longitudinal & & \\
\hline Estudos quanti-qualitativos & 1 & 2,5 \\
\hline Estudo epidemiológico, transversal & 2 & 5,0 \\
\hline Observacional & 1 & 2,5 \\
\hline Transversal, descritivo e exploratório & 1 & 2,5 \\
\hline Retrospectivo & 2 & 5,0 \\
\hline Prospectivo & 5 & 12,5 \\
\hline Revisões: integrativa e sistemática & 40 & 100,0 \\
\hline Total & & \\
\hline
\end{tabular}

Tabela 4. Produção científica de centro cirúrgico, por objeto de estudo.

\begin{tabular}{|l|c|c|}
\hline \multirow{2}{*}{ Objeto de estudo } & \multicolumn{2}{|c|}{ Total } \\
\cline { 2 - 3 } & N & $\%$ \\
\hline Assistência transoperatória & 24 & 60,0 \\
\hline Saúde do trabalhador & 10 & 25,0 \\
\hline Custos & 03 & 7,5 \\
\hline Gestão & 03 & 7,5 \\
\hline Total & 40 & 100,0 \\
\hline
\end{tabular}




\section{DISCUSSÃO}

No período de 2005 a 2016, ocorreram 13 publicações de TD, representando $32,5 \%$ das produções científicas do total desse período, destacando-se o ano de 2014, com o maior número de publicações (7,5\%). Quanto às DMA, em todo o período, obtiveram-se publicações sobre a temática CC, realçando-se os anos de 2009 e 2013, com 12,5 e 15,0\%, respectivamente.

Quando comparados com relação ao número de produções acadêmicas entre DMA e TD, evidencia-se um predomínio de publicação em CC dos programas de mestrado acadêmico (67,5\%). Esse fato pode ser explicado tanto pelo quantitativo expressivo de cursos de mestrado existentes no Brasil como pelo tempo de elaboração e defesa das dissertações ser inferior ao das teses, favorecendo, assim, as publicações em um período mais curto. Tal resultado corrobora outro estudo bibliométrico, no qual se observou a prevalência nas produções de DMA em relação às produções de $\mathrm{TD}^{10}$.

Entre as instituições de ensino representadas pela amostra analisada neste estudo bibliométrico, a maioria das universidades está localizada na região Sudeste ${ }^{10}$. Esse resultado pode ser justificado pelo fato de que todas as universidades com produção científica significativa ou expressiva compõem o ranking das 50 melhores universidades do Brasil, exceto a USP, pois essa instituição não participa do Exame Nacional de Desempenho dos Estudantes (Enade), prova que é utilizada como base para elaboração da nota ${ }^{11}$.

Entretanto há predomínio das produções científicas da USP (32,5\%) e da USP-RP (25,0\%). A USP é uma instituição pública, mantida pelo estado de São Paulo e ligada à Secretaria de Desenvolvimento Econômico, Ciência, Tecnologia e Inovação (SDECTI). Entre as universidades públicas brasileiras, é aquela com o maior número de vagas de graduação e pós-graduação, sendo responsável também pela formação do maior número de mestres e doutores do mundo, bem como responsável por metade de toda a produção científica do estado de São Paulo e mais de 25,0\% da produção brasileira ${ }^{12}$.

Quanto às abordagens metodológicas encontradas nas produções acadêmicas estudadas, a mais frequente foi a quantitativa (45\%), seguida da qualitativa (30\%), corroborando outra investigação bibliométrica. Percebe-se que há tendência à realização de estudos com a primeira, por permitir verificação estatística entre variáveis e possível generalização; em contrapartida, os métodos qualitativos centram-se mais nos padrões que dão significado aos fenômenos e que contribuem para a compreensão da experiência como um todo'.

Também se observa a prevalência de estudos qualitativos nos bibliométricos. Assim, evidencia-se certa divisão pelas duas metodologias, pois, se, por um lado, há necessidade de quantificar, por meio da estatística, determinados fenômenos, por outro, é fundamental ter compreensão de aspectos não quantificáveis, que permitam a compreensão da experiência de uma forma mais humanizada, o que apenas a metodologia qualitativa permite ${ }^{9,10,13}$.

Entende-se, no entanto, que os avanços tecnológicos em cirurgia, a complexidade do cuidado e o estado vulnerável do paciente cirúrgico exigem que a atuação do enfermeiro no CC seja respaldada em conhecimentos evidentes de atuação e de concepção de que o alvo é a realização de assistência perioperatória para o sucesso do procedimento anestésico-cirúrgico com segurança ${ }^{14}$. Ao considerar o percentual de publicações sobre a assistência transoperatória (60\%), é possível inferir que tal tema é de suma importância para busca da melhoria da assistência ao paciente cirúrgico.

Analisando os objetos de estudo de cada produção selecionada para o presente, destaca-se o tema envolvendo assistência transoperatória, com assuntos sobre humanização na prática dos cuidados de enfermagem, prevenção de infecção de sítio cirúrgico, segurança do paciente, dinâmica dos cuidados de enfermagem transoperatória, hipotermia, diagnóstico de enfermagem, sistematização da assistência de enfermagem, posicionamento cirúrgico, antibioticoprofilaxia e complicações transoperatórias.

Entendendo a maior representatividade da categoria temática "assistência ao paciente", de acordo com os resultados encontrados, esta tem seu destaque por abordar questões sobre a prática assistencial da equipe de enfermagem, visando à segurança do paciente, correlacionando-a a preocupação do segundo desafio global da Organização Mundial da Saúde (OMS), "Cirurgias seguras salvam vidas". Esse programa visa melhorar a segurança da assistência cirúrgica no mundo, por meio da definição de um conjunto central de padrões de segurança que possam ser aplicados em todos os países e cenários ${ }^{15,16}$.

Dessa forma, a assistência cirúrgica tem sido um componente essencial ao cuidado à saúde, tendo sua necessidade ampliada com a demanda cada vez maior de intervenções cirúrgicas, devido a doenças cardiovasculares, neoplasias e 
traumas, decorrente do aumento da expectativa de vida e da violência ${ }^{1,15}$.

Apesar do estímulo de produção nessa área por alguns órgãos internacionais e nacionais e do crescente número de procedimentos cirúrgicos da população brasileira, o presente estudo evidenciou baixa produção científica com a temática CC por enfermeiros, especialmente no período intraoperatório, área de extrema relevância para atuação do enfermeiro.

O uso dos recursos disponíveis nas bases de dados contribuiu para a realização deste estudo, fato importante para a profissão de enfermagem, pois colabora para divulgação do conhecimento e desenvolvimento da categoria. Todavia, teve-se como limitação a coleta de dados na base da CEPEn, visto que não foi possível realizar a busca nos anos de $2006 \mathrm{e}$ 2007, por indisponibilidade dos catálogos. Ademais, outro fator que dificultou a realização da pesquisa foi o fato de as teses e dissertações serem agrupadas em catálogos disponíveis por ano, o que não permite o filtro por descritores.

\section{CONCLUSÃO}

Este estudo permitiu identificar a produção científica acadêmica sobre CC por enfermeiros que concluíram mestrados acadêmicos e doutorados, além de descrever os tipos de abordagens metodológicas e seus principais objetos estudados. Diante disso, observou-se, nos últimos 11 anos, uma produção inconstante por parte dos enfermeiros sobre esse assunto.

A pesquisa contribuiu ainda para a identificação dos objetos de estudo e metodologias mais utilizadas pelos enfermeiros na área de CC, o que poderá ser significativo na orientação da escolha de futuras produções científicas. Considera-se que ainda há necessidade de maior investimento em quantidade $\mathrm{e}$ qualidade em produção científica, de forma que se enfatize a importância da assistência de enfermagem transoperatória.

Com essa análise bibliométrica, será possível configurar um panorama relevante para a enfermagem, demonstrando padrões de pesquisa e identificação de tendências para futuras produções científicas acadêmicas.

\section{REFERÊNCIAS}

1. Carvalho R, Bianchi ERF, eds. Enfermagem em centro cirúrgico e recuperação. $2^{\mathrm{a}}$ ed. Barueri: Manole; 2016.

2. Possari JF. Centro cirúrgico: planejamento, organização e gestão. $4^{\mathrm{a}}$ ed. São Paulo: latria; 2009

3. Associação Brasileira de Enfermeiros de Centro Cirúrgico, Recuperação Anestésica e Centro de Material e Esterilização (SOBECC). Diretrizes de práticas em enfermagem cirúrgica e processamento de produtos para a saúde. 7ª ed. São Paulo: SOBECC/Barueri: Manole; 2017.

4. Carvalho R, ed. Enfermagem em centro cirúrgico e recuperação anestésica. Manuais de Especialização Einstein. Barueri: Manole; 2015.

5. Cruz EA, Soares E. A tecnologia em centro cirúrgico e o processo de trabalho do enfermeiro. Esc. Anna Nery R Enferm. 2004;8(1):109-15.

6. Bieging P, Busarello RI, Aquino V. Perspectivas na comunicação: audiovisual, publicidade e rede social. São Paulo: Pimenta Cultural; 2016.

7. Areias FZ, Abrão FMS, Moura LF, Silva RCL, Costa SFG, Silva CRL. Análise bibliométrica de artigos sobre laringectomia total. Rev Bras Enferm. 2016;30(3):1-11. http://dx.doi.org/10.18471/rbe.v30i3.15790

8. Alencar MSM, Bochner R, Dias MFF, Antunes MAS. Análise de produção científica brasileira sobre nanotecnologia e saúde. Rev Eletron Comum Inf Inov Saúde [Internet]. 2017 [acessado em 4 nov. 2017];11(1). Disponível em: https://www.reciis.icict.fiocruz.br/index.php/reciis/article/view/1199 http://dx.doi.org/10.3395/reciis.v11i1.1199

9. Ferreira MAL, Pereira AMNA, Martins JCA, Figueiredo MCB. Cuidados paliativos e enfermagem nas dissertações e teses em Portugal: um estudo bibliométrico. Rev Esc Enferm USP. 2016;50(2):313-19. http:// dx.doi.org/10.1590/S0080-623420160000200019

10. Spindola T, Pimentel MRRA, Barros AS, Franco VQ, Ferreira LEM. Produção de conhecimento acerca das doenças sexualmente transmissíveis na população jovem: pesquisa bibliométrica. J Res Fundam Care Online [Internet]. 2015 [acessado em 22 nov. 2017];7(3):3037-49. Disponível em: http://dx.doi.org/10.9789/2175-5361.2015.v7i3.3037-3049

11. Brasil. Ministério da Educação. Enade - apresentação [Internet]. [acessado em 8 dez. 2017]. Disponível em: http://portal.mec.gov.br/enade

12. Brasil. Fundação CAPES. Brasília: Ministério da Educação; 2009 [acessado em 8 dez. 2017]. Disponível em: http://www.capes.gov.br/

13. Baggio MA, Rodrigues MA, Erdmann AL, Figueiredo MCAB, Vieira MMS. Produções de teses e dissertações da enfermagem em Portugal de 20002010: estudo bibliométrico. Texto Contexto Enferm. 2014:23(2):25060. http://dx.doi.org/10.1590/0104-07072014002190012

14. Turrini RNT, CostaALS, Peniche ACG, Bianchi ERF, Cianciarullo TI. Ensino de enfermagem em centro cirúrgico: transformações da disciplina na Escola de Enfermagem da USP (Brasil). Rev Esc Enferm USP. 2012;46(5):126873. http://dx.doi.org/10.1590/S0080-62342012000500032

15. Brasil. Ministério da Saúde. Agência Nacional de Vigilância Sanitária. Organização Mundial da Saúde. Segundo desafio global para a segurança do paciente. Cirurgias seguras salvam vidas. Rio de Janeiro: Organização Pan-Americana de Saúde; 2009.

16. Gawande A. Checklist: como fazer as coisas bem-feitas. Rio de Janeiro: Sextante; 2011. 